\title{
PREFERRED FOLLOWING DISTANCE AND PERFORMANCE IN AN EMERGENCY EVENT WHILE USING COOPERATIVE ADAPTIVE CRUISE CONTROL
}

\author{
Stacy A. Balk ${ }^{1}$, Steven Jackson ${ }^{1}$, and Brian Philips ${ }^{2}$ \\ ${ }^{1}$ Leidos, Transportation Solutions and Technology Applications Division \\ ${ }^{2}$ Federal Highway Administration, US Department of Transportation \\ E-mail: stacy.balk.ctr@dot.gov
}

\begin{abstract}
Summary: This study explored human factors issues associated with cooperative adaptive cruise control (CACC); specifically the relationship between drivers' preferred following distance, assigned following distance, and driving performance. Participants drove in a dedicated lane and experienced a vehicle merging in front of their vehicle and later, an emergency event that required intervention in order to avoid a collision. Drivers followed at either a near or a far distance. Drivers' perceived workload did not vary between the cruise and postmerge periods. However, workload was significantly greater after the emergency crash event. Workload did not vary significantly based on following distance assignment or preference. Those participants assigned to the near following distance were more likely to hover their foot over the brake during the merging event and to react faster to the emergency event. As with workload, performance (collision avoidance) did not vary significantly due to following distance assignment or preference. In other words, one's abilities may not necessarily reflect their following preferences. This is a promising finding for widespread implementation of CACC.
\end{abstract}

\section{INTRODUCTION}

CACC combines two driver assist systems: (1) adaptive cruise control, which uses radar or light detection and ranging (LIDAR) sensors to automatically maintain a gap the driver has selected between the driver's vehicle and a slower moving vehicle ahead, and (2) dedicated short-range communications (DSRC) to transmit and receive data with surrounding vehicles so that the cruise control system can more quickly respond to changes in the speed and location of other CACC vehicles, even vehicles that are not in view of the driver (Jones, 2013).

When using CACC, drivers share vehicle control with an automated system that includes vehicle-to-vehicle (V2V) and vehicle-to-infrastructure (V2I) communications. Communications between nearby CACC equipped vehicles will enable automated coordination and adjustment of longitudinal control through throttle and brake activations. Automated control should enable CACC equipped vehicles to safely travel with smaller gaps between vehicles than drivers could safely manage on their own. Smaller gaps should subsequently increase the roadway capacity without increasing the physical amount of roadway. However, shorter following gaps lead to problematic human factors issues.

At $65 \mathrm{mph}$ a one second gap leaves approximately $95 \mathrm{ft}$ between vehicles. Previous studies have shown that drivers feel both comfortable and safe travelling at gaps shorter than one second. For example, in an on-road study testing drivers' choices in following distances, drivers regularly used gap settings shorter than one second. In fact, overall when following another vehicle, 
drivers elected to set the gap at $0.7 \mathrm{~s}$ or $0.6 \mathrm{~s}$ eighty percent of the time (Nowakowski, et al., 2010). However, with a $0.6 \mathrm{~s}$ gap, there is only approximately $57 \mathrm{ft}$ between vehicles (at 65 $\mathrm{mph}$ ). If an average vehicle length is assumed to be around $20 \mathrm{ft}$, this leaves less than $18.5 \mathrm{ft}$ of buffer in either direction for a merging vehicle. As a result, at these shorter distances, drivers may not feel comfortable merging or having a vehicle merge in front of them in a CACC platoon. Furthermore, gap-based discomfort may vary from person to person. It is possible that individual differences in preferred following time gap may influence performance (i.e., reaction time or maneuver) in the event that the driver needs to overtake the CACC system and regain manual control of the vehicle. For this reason, preferred following distance as it relates to performance is considered.

Another very important assumption made in this study is that the CACC system will require dedicated infrastructure in its early implementation. This infrastructure requires that the CACC lane (or lanes) be physically separated from "normal" travel. This is important for several reasons. CACC may be the most beneficial in congested regions. This congestion often leads to lower travelling speeds and a great deal of speed variation (i.e., stop-and-go or slow-and-go traffic). Because CACC equipped vehicles travelling in a separate lane will travel at fairly constant speeds with standard gap distances, the lane will be less susceptible to speed variability. As a result, vehicles in the CACC lane are likely to be travelling at speeds greater than the "normal" travel lanes. The speed differential between the two types of lanes will introduce problems reaching speeds great enough to transfer from one type of lane to the other. Instead, drivers will be required to enter the lane from a separate onramp - much like drivers entering and exiting dedicated HOV lanes. The physical separation between the two types of lanes prevents non-CACC equipped vehicles from entering the CACC lane and disrupting travel flow stability. In order to mimic this anticipated initial early implementation of CACC, participants drove in a dedicated and physically separated lane.

Driver performance remains important in semi-autonomous systems such as CACC. CACC systems do not maintain lateral control of the vehicle, and braking is not always the best or safest response to a slower or stopped vehicle ahead. This can be especially problematic in the case of system failure or an emergency event (e.g., a crash upstream). This study explored driver performance while using CACC. The goal of this research was to address some of the critical human factors issues for CACC usage related to the abilities and limitations of the drivers using the system. In a previous experiment it was found that the CACC system was effective in preventing crashes and workload was rated as low. However, the gap (time-gap, from front bumper of host to rear bumper of preceding vehicle) was $1.1 \mathrm{~s}$. For a CACC system to greatly increase highway capacity, it would need to maintain smaller gaps. Such a requirement raises several questions regarding (a) driver acceptance of smaller gaps, (b) the effect of following distance preference on crash avoidance, and (c) the effect of this preference on perceived workload.

As previously noted, many drivers already accept gaps smaller than $1.1 \mathrm{~s}$. For instance, TaiebMaimon \& Shinar (2001) reported a study in which the perceived minimum safe gaps were $0.7 \mathrm{~s}$ or less and comfortable perceived gaps were less than 1s. It is possible, and likely, that acceptable gap perception varies greatly between drivers and driving environments. The present work explores gap acceptability and driving performance in a driving simulator. 
The goals of this study were:

1. Assess drivers' workload under two different CACC following gaps (near and far).

2. Assess drivers' reaction to a vehicle merging in front of them under different following gaps.

3. Assess drivers' reaction to an emergency event that requires driver intervention to avoid collision.

4. Determine if preferred time gap following distance affects 1-3.

\section{METHOD}

The National Advanced Driving Simulator (NADS) 1/4 cab miniSim was used. The simulator uses three 42" screens to display the forward roadway, side and rearview mirrors. An additional 12 in widescreen screen is used to display dashboard information. A 7 in LCD touch-screen was mounted to the right of the steering wheel (near where a center console would be located) and served as the CACC system display. For all conditions, the screen displayed the vehicle set speed, the set following distance, and the status of the CACC system. The engage button on the right side of the display was used by the participants to engage CACC.

\section{The Simulation Scenario}

Preferred Gap Determination. Participants drove in a dedicated center lane on a simulated 8-lane interstate highway (4 lanes in each direction). Entrance to the center dedicated-lane was accessed from the left side of the roadway from a ramp and was separated from other lanes by a jersey barrier. The environment was similar to suburban-rural interstate driving with a mix of trees and buildings along the roadway.

The simulation began with the participant vehicle as the only vehicle on the roadway. This time period was provided for participants to become accustomed to the feel of the driving simulatorincluding the steering, acceleration, and braking capabilities. After a few minutes the participant came upon another vehicle. That vehicle acted as a lead vehicle and drove at 55, 65, 70, and 55 mph for three minutes each. The participant first followed the vehicles at a comfortable distance and then repeated the drive at a distance considered to be the minimum safe distance. The time gap from the second drive was used to determine if a participant was a near or a far follower (described in more detail below).

Assigned Gap Drive. The participants began stopped on an onramp in the third position of a four car platoon. As the scenario began and the ramp meter turned green, the platoon proceeded down the ramp and accelerated to $70 \mathrm{mph}$ while maintaining the appropriate gap $(0.6$ or $1.1 \mathrm{~s}-$ described in more detail later). Approximately five minutes into the drive another CACC vehicle merged into the platoon directly in front of the participant and halfway between the participant's vehicle and the vehicle the participant had been following. The CACC system adjusted the gaps of the affected vehicles back to the assigned gap. If the participant braked in this situation, the CACC system disengaged and needed to be re-engaged.

At approximately 20 minutes into the drive, a vehicle speeded down an onramp, merged in front of the platoon and crashed. (The crash was animated, but was not in the participant's line of sight if driving centered in the travel lane.) The "crash avoidance" event began when the lead vehicle 
in the platoon decelerated at $32 \mathrm{ft} / \mathrm{s}^{2}(1 \mathrm{~g})$. One tenth of a second after the lead vehicle began braking; all of the CACC vehicles behind it simultaneously began to decelerate at $0.4 \mathrm{~g}$ and illuminated their brake lights. The engine noise was configured to exaggerate the engine RPM in order to more adequately cue $0.4 \mathrm{~g}$ deceleration.

\section{Gap Assignment}

Assignment to gap groups was based on mean gap maintained during the second drive and the median following distance identified from previous work $(0.91 \mathrm{~s})$. Participants were assigned to near or far preference groups based on whether their preferred gap fell below or above the median, respectively. Next, participants were assigned to either a congruent or incongruent following preference group. Of the 59 drivers with a preferred near following distance, 29 were assigned to the congruent (near, $0.6 \mathrm{~s}$ ) gap in drive 3, and 30 were assigned to the incongruent (far, $1.1 \mathrm{~s}$ ) gap. Of the 39 drivers with a preferred far following distance, 20 were assigned to the congruent (far, $1.1 \mathrm{~s}$ ) gap in drive 3, and 19 were assigned to the incongruent (near, $0.6 \mathrm{~s}$ ) gap in drive 3.

\section{Workload Assessment}

Driver workload was assessed by administration of the National Aeronautics and Space Administration Task Load Index (NASA-TLX), and was measured three times. The first assessment was approximately 5 min into the experimental drive, after the vehicle merged in front of the participant at about $30 \mathrm{~s}$ after the platoon had stabilized. The second assessment was administered approximately 10 min into the drive and was intended to assess the workload associated with driving in a stable, unchanging cruising state; that is a baseline index. At this point, drivers were between merging events and likely to feel comfortable with the driving task in general. The third and final NASA-TLX was administered immediately after the final collision avoidance event.

\section{Participants}

Participants were 98 (49 male) licensed drivers recruited from the Washington, DC metropolitan area. Participants were required to be at least 18 years of age, had a valid driver's license, and were screened for susceptibility to motion and simulator sickness. Age ranged from 21 to 73 years $(\mathrm{M}=43.3$; median 43.5 years $)$. Data from five participants were excluded from analyses due to corrupt data files.

\section{Procedure}

After providing informed consent and receiving a high level overview, participants completed the first two drives (meant to familiarize the participant to the simulator and determine following distance preference). After the completion of the second drive, a slideshow presentation was shown to all participants. The presentation provided an overview of the experimental instructions and familiarized participants with the NASA-TLX questions. Videos explaining the functionality of CACC were also shown.

The use of the multifunction LCD display was explained and demonstrated as necessary. Next, participants drove the third scenario while using the CACC system. If at any time the participant used the brake, or otherwise disengaged the CACC, he or she was reminded to use the multifunction display to reengage the system. Participants were provided time to ask any 
questions about the study, debriefed, thanked and paid for their time. In total, participation lasted 60-90 minutes.

\section{RESULTS}

Workload. The NASA-TLX was administered verbally at three points during the third drive: Shortly after the first merge, during a cruise period, and after the crash event. The effects of CACC following distance on workload were tested using generalized estimating equations (normal response distribution, identity link function) with NASA-TLX as a repeated measure and experimental treatment conditions (preferred gap and assigned following gap) as the between-group factors of interest. As expected, the location of the NASA-TLX assessment significantly affected perceived workload, $\chi^{2}(2)=129.81, p<0.001$. The mean NASA-TLX score after the first merge $(M=15.76)$ was not significantly different than during the cruise period $(M=13.11)$. However, mean workload was significantly greater after the crash event $(M$ $=55.44)$ than at the two previous times.

Merging vehicle response. In order to assess trust and comfort in the CACC system, participant response to the merging event was evaluated. After approximately five minutes of driving, a vehicle traveled down an onramp and merged directly in front of the participant vehicle. The CACC system was programmed in such a way that it was not necessary to interfere with, or override, the vehicle speed during the merge. Nonetheless, three older participants (two males) pressed the brake pedal. Because only three participants pressed the brake pedal during this merge event, braking was not further analyzed. Despite few participants using the brake pedal, the speed and short distance between the participant vehicle and the merging vehicle may have made the driver uncomfortable. As a result, foot position immediately prior to, and during, the vehicle merge was explored.

Foot pedal video data were coded beginning at $30 \mathrm{~s}$ immediately preceding the merge event. The participant's foot position was noted. Foot movement to the brake pedal into a hovering position in anticipation of the merging vehicle was coded. Those participants following at the near distance were significantly more likely to hover over the brake pedal than those at the longer distance, $\chi^{2}(1)=5.27, p=0.022$. This is not surprising given the short distance between the participant vehicle and the merging vehicle, which was likely to generate some mild discomfort in following distance. Participants appeared to be readying themselves to apply the brake if necessary.

No difference in foot hovering over the brake was found based on preferred following distance, $\chi^{2}(1)=0.29, p>0.05$. Further, no significant interaction between preferred following distance and assigned following distance was found, $\chi^{2}(1)=0.00, p>0.05$. In other words, those preferring to drive at a shorter following distance were just as likely to hover their foot over the brake pedal as those that preferred a longer following distance.

Crash event reaction. Next, participant reaction time for those that used the brake pedal (all but three) was explored. Participant reaction time was calculated as the time between when the merging vehicle entered the participant's lane and when the participant first depressed the brake pedal. Participants that drove at the close distance $(\mathrm{M}=2.77 \mathrm{~s})$ depressed the brake pedal significantly sooner than the participants that drove at the far distance $(\mathrm{M}=4.88 \mathrm{~s}), \chi^{2}(1)=$ 
14.34, $p<0.001$ (see Figure 1). No difference in reaction time based on preferred following distance was found, $\chi^{2}(1)=0.00, p>0.05$. Similarly, no interaction between preferred and assigned following distance was found, $\chi^{2}(1)=1.08, p>0.05$.

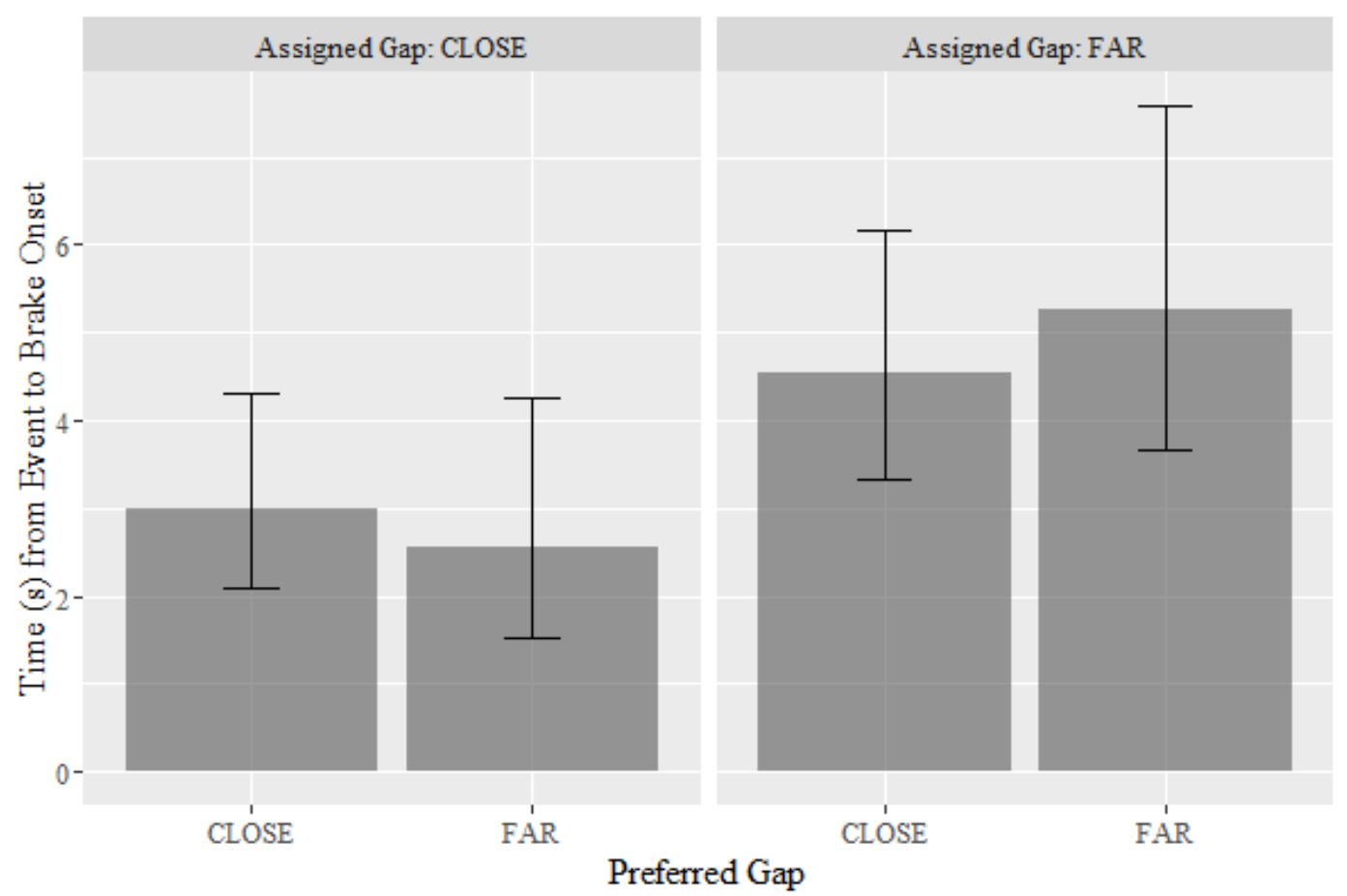

Figure 1. Time (s) from principal other vehicle entering the traffic flow to participant brake pedal onset based on preferred and assigned time gaps.

Another manner in which brake pedal response can be examined is through maximum pedal depression. The time from initial brake pedal activation until the maximum pedal depression was explored. Preferred following distance approached significance in an interesting way, $\chi^{2}(1)=$ $3.54, p=0.059$. Those participants that preferred to follow at a far distance, reached maximum pedal depression $(\mathrm{M}=1.28 \mathrm{~s})$ faster than those that preferred to follow at a near following distance $(\mathrm{M}=2.05 \mathrm{~s})$. While the difference is not significant, the trend shows that those drivers that are more comfortable following at a greater distance may be more likely to react with full brake pedal force more rapidly. No significant difference in assigned following distance was found, $\chi^{2}(1)=0.69, p>0.05$. Similarly, the interaction between preferred and assigned following gap was not significant, $\chi^{2}(1)=0.82, p>0.05$.

Crashes. Next, participant crashes during the final event were examined. A crash was defined as the participant vehicle colliding with the immediately preceding vehicle. Participants who drove at the close distance experienced significantly more crashes $(\mathrm{M}=0.82)$ than the participants that drove at the far distance $(\mathrm{M}=0.61), \chi^{2}(1)=4.32, p=0.038$. No difference in collision rate based on preferred following distance was found, $\chi^{2}(1)=0.49, p>0.05$. Similarly, no interaction between preferred and assigned following distance was found, $\chi^{2}(1)=1.27, p>0.05$. 


\section{DISCUSSION}

Participants drove a scenario with CACC engaged with either a near $(0.6 \mathrm{~s})$ or far $(1.1 \mathrm{~s})$ time gap. This gap was either congruent or incongruent (as determined by preliminary drives) with preferred following distance. During that drive, a vehicle merged directly in front of the driver. Later a vehicle, out of direct line of sight, crashed which required the driver to take action in order to avoid collision. Driver performance during these two events and perceived workload were assessed.

As one might expect, drivers' perceived workload (as assessed by the NASA-TLX) varied by the context in which it was administered (cruise, post-merge, post-crash). Not surprisingly, workload was the greatest after the crash event. However, no significant variation between the workload after the initial merge event and a relaxed cruise time period was found. This suggests that participants trusted the CACC system to allow sufficient space for a merging vehicle.

At the first vehicle merge event, only three people depressed the brake. While this highlights an overall trust in the system, foot hovering behavior highlighted that those people following at the near CACC setting were prepared to override the system if necessary as the other vehicle merged. This was independent of preferred following distance. That is, those that prefer to follow at closer time gaps were as likely to hover their foot over the brake pedal in anticipation of potentially overriding the CACC system as those that prefer to follow at longer time gaps.

During the crash event, those participants assigned to the closer following distance both reacted sooner and experienced more crashes, regardless of following distance preference. Given that participants following closer had physically less distance to react in order to avoid a collision, it is not surprising that more of these participants experienced a collision.

Throughout the study, participants' following distance preference did not affect performance. In other words, one's following preferences may not necessarily be a reflection of their driving abilities. This is a promising finding for widespread implementation of CACC. While overall comfort level may vary across drivers, these findings support the idea that performance will depend more on overall CACC following distance settings than with drivers' personal preferences. This will allow CACC systems to implement a single following distance gap (or continuum of gaps based on vehicle physics). These results also highlight the need to implement well designed human factors centric systems that clearly indicate to drivers when it is necessary to take over control of the vehicle. Without such measures, it is possible that CACC implementation may not result in roadway safety improvements.

\section{ACKNOWLEDGEMENT}

This research was sponsored by the FHWA Exploratory Advanced Research Program under contract DTFH61-13-D-00024.

\section{REFERENCES}

Jones, S. (2013). Cooperative Adaptive Cruise Control: Human Factors Analysis, FHWA-HRT13-045. Federal Highway Administration, Washington, DC.

Nowakowski, C., et al. (2010). Coorperative Adaptive Cruise Control: Testing Drivers' Choices of Following Distances. 